\title{
EXPERIÊNCIA DE PRODUÇÃO DE RECURSOS PEDAGÓGICOS ABERTOS EM LABORATÓRIO \\ DE INOVAÇÃO CIDADÃ - LABIC: \\ UMA ANÁLISE DO COMUM
}

\author{
RENATO FROSCH
}

\section{RESUMO}

Este artigo apresenta a experiência do autor no desenvolvimento de material pedagógico para crianças com deficiência visual, apoiado por hardware aberto (impressora 3D), pelos princípios da cultura maker e da ciência aberta. A etapa do projeto abordada neste trabalho foi realizada por uma equipe multidisciplinar no Laboratório de Inovação Cidadã - LabicAR, com duração de 15 dias, em outubro de 2018, em Rosário - Argentina. A abordagem metodológica foi desenvolvida por um processo aberto e democrático, incluindo a seleção dos membros da equipe e o projeto/ protótipo executado. Os resultados alcançados no laboratório apontam para possibilidades de disseminação e aprimoramento da criação e popularização de material pedagógico, como softwares (principalmente aqueles relacionados à modelagem digital) e hardware em amplo uso livre, a fim de obter aplicações progressivas para promover, neste caso, o aumento da aprendizagem escolar para um público específico: comunidades de aprendizagem incluindo crianças com deficiência visual.

Palavras-chave: cultura maker, ciência aberta, modelagem 3D.

\begin{abstract}
This article presents the author's experience in developing pedagogical material for children with visual disabilities, supported by $3 \mathrm{~d}$ printer and the principles of maker culture. The stage of the project addressed in this paper was carried out by a multidisci-
\end{abstract}


plinary team in the Laboratory of Citizen Innovation - LabicAR, lasting 15 days on October 2018, in Rosario - Argentina. The methodology approach have been chosen by an openness and democratic process including the team members and the project to be carried out. The results achieved in the laboratory so far point out to possibilities of dissemination and improvement of the creation and popularization of pedagogical material such as softwares (mainly those related to digital modeling) and hardware in wide free use in order to obtain a progressive applications to promote, in this case, school learning increase for a specific public: children with visual disabilities.

Keywords: maker culture, open science, $3 \mathrm{~d}$ modeling.

\section{INTRODUÇÃO}

A educação especial é comumente apoiada pela produção de material pedagógico utilizando predominantemente técnicas artesanais, principalmente aquelas voltadas para a educação de crianças com deficiência visual. Devido às suas características esse tipo de produção aponta para pouca repetibilidade e também exige largo tempo de criação, levando aos educadores um distanciamento do foco de sua prática em sala de aula e das reflexões dos processos cognitivos propriamente relacionados à aprendizagem da educação básica.

Por outro lado, os resultados alcançados em experiências que utilizam recursos digitais ou prototipagem rápida aparecem como oportunidades de meios de produção democrática e contemporânea do recurso pedagógico, sendo (1) universal, (2) aberto, (3) e replicável. Detalhamos os 3 itens: (1) universal, porque pode ser um material para todos: pessoas com ou sem deficiências visuais e outras limitações de linguagem, como indivíduos com diferentes modos de ver e de se relacionar com modelos pedagógicos físicos que são baseados em recursos teóricos de design universal; (2) aberto, devido ao compartilhamento digital, chamado open source, cujos códigos, modelagem e protocolos são 
ferramentas gratuitas, incluindo também sites que podem ser acessados e usados com licenças abertas, como creative commons; e, finalmente (3) replicável, já que o mesmo modelo pode ser impresso em $3 \mathrm{~d}$ muitas vezes com adaptações ou utilizando o modelo primário idêntico disponível digitalmente, garantindo assim maior quantidade de utilizações.

\section{PROCESSOS DE PRODUÇÃO DE MATERIAL PEDAGÓGICO EM UM LABORATÓRIO DE INOVAÇÃO CIDADÃ}

O produto material pedagógico foi o resultado de um complexo processo humano e tecnológico estabelecido, cocriado e apoiado pelas práticas que ocorreram no Laboratório de Inovação Cidadã - LABIC, realizado em Rosário, Argentina, em 2018.

Os LABICs nascem de modo mais enfático no começo dos anos 2000, principalmente, com o surgimento do Media-Lab Prado - MLP, em Madrid. Lafuente \& Estalella (2014) caracterizam e realçam relações entre comum, produção cultural e tecnologia que são elementos estruturantes deste tipo de laboratório de inovação cidadã:

(...) un proyecto donde el procomún adopta la condición de objeto epistémico para la experimentación y señalamos la extendida presencia del procomún en el ámbito de la producción cultural y creativa en España (...) situamos la emergencia del debate sobre el procomún en el entorno digital y señalamos la emergencia del procomún urbano como objeto de interés y argumento para la crítica al neoliberalismo desde proyectos activistas que toman al procomún como un concepto clave (...). Lafuente \& Estalella (2014, p.3).

As descrições dos itens a seguir apontam e detalham esse processo da ciência aberta em laboratórios de inovação.

\section{A. Os Laboratórios de Inovação Cidadã}

Os laboratórios de inovação cidadã são espaços onde qualquer cidadão pode participar e colaborar com outros, com- 
partilhando conhecimentos, ideias e experiências para cocriar projetos que possam trazer propostas de soluções para melhorar qualquer tipo de situação a partir da mais diversificada fonte de problemas.

O posicionamento epistemológico que incorpora o saber leigo ao lado do acadêmico tem sido pensado por Lafuente \& Estalella (2015) como a constituição de uma ciência comum que se faz não só com conhecimentos de diferentes procedências, mas entre todos. Uma ciência diversa da ciência pública e da privada, pois seu objetivo não é chegar rapidamente a resultados pela expertise de acadêmicos, mas considerar na mesma medida no processo de construção do conhecimento a experiência dos cidadãos, qual seja: a do enfermo sobre seu padecer, a dos camponeses sobre seu território, a dos vizinhos sobre seu bairro.

Lafuente \& Estalella (2015) avançam na proposta de uma ciência comum não apenas como um bem comum disponível, mas principalmente como algo que se constrói colaborativamente entre todos. Os autores destacam que os cidadãos participem de debates estratégicos para a vida contemporânea.

É possível, de acordo com Lafuente \& Estalella (2015), imaginar que a pesquisa cidadã possa se contrapor aos dados oficiais e influenciar na investigação. Do ponto de vista dos autores, podemos estar no alvorecer de um novo regime epistêmico que venha a incluir outros saberes, com novos atores e diferentes perguntas. Para incorporar outros saberes, a ciência comum necessita de outro tempo, mais lento, para escutar e dialogar. Precisa também de outras formas de validação que reconheçam os saberes não acadêmicos, já que não exige credenciais para a participação.

Neste sentido, os espaços maker, laboratórios de fabricação digital geralmente compostos por equipamentos como impressoras $3 \mathrm{~d}$, cortadoras laser, máquinas $\mathrm{CNC}$, se colocam de forma qualificada, conceitualmente, para promover elementos indutores em contribuições para processos sociais e políticos contemporâneos. 
A lógica propiciada pelos espaços maker confronta o modelo atual de produção de objetos iguais, de produção intencionalmente seriada. A fabricação digital pode produzir protótipos e outros desenvolvimentos tecnológicos não comerciais, ou seja, que atendam a pequenos grupos de sujeitos ou um ator social específico. Gershenfeld (2005) realça esta colocação:

(...) mas se o mercado é apenas uma pessoa, então, o protótipo é o produto. As grandes máquinas continuarão a produzir em massa coisas usadas em grandes quantidades; porcas e parafusos são valiosos porque eles são idênticos em vez de únicos. Mas pequenas máquinas vão customizar produtos que dependam da diferença e da customização (...). Gershenfeld (2005, p.7).

Para isso, os sujeitos envolvidos na fabricação digital maker utilizam preferencialmente a experiência, os conhecimentos adquiridos em diversas trajetórias formativas, sejam formais - alcançadas em instituições de ensino e outros formatos institucionalizados, ou não formais - em trajetórias de experiências diversas como vivências, saberes tradicionais e oportunidades de construção de conhecimentos tácitos.

Nessa perspectiva, Samagaia e Delizoicov Neto (2015) destacam que os conhecimentos e habilidades, que são assim sistematicamente ampliados, testados e melhorados, concebidos prioritariamente na forma de recursos abertos, constituem geralmente uma base de trabalho compartilhada, de usufruto gratuito e coletivo e facilmente acessível. Os sujeitos makers identificam-se ainda a um movimento organizado, estruturado a partir da noção de mínimos recursos e máxima partilha de ideias, de projetos e de concepções.

Considerando o formato apresentado, é importante dizer que existem outras discussões científicas que tratam de formas inovadoras e disruptivas de gestão do conhecimento apoiada em elementos tecnológicos, mas não serão objeto deste trabalho. 
Indo adiante, há uma expectativa dos organizadores de laboratórios cidadãos de que o trabalho possa ocorrer de forma colaborativa, criando projetos que discutam e evoluam temáticas como transparência, participação cidadã, soluções tecnológicas para problemas específicos, novos temas de redes sociais, étnicas ou culturais, ecologia e assim por diante. No entanto, vale a pena ressaltar que os cidadãos têm participação direta e voluntária no laboratório e podem candidatar-se a trabalhar nele por meio de uma sessão de autoapresentação de suas habilidades e competências, independentemente de sua formação. Esses cidadãos selecionados serão aqueles que desenvolverão e implementarão propostas ou protótipos que podem eventualmente promover mudanças sociais, aumentando o conhecimento dentro de um movimento importante que tem sido chamado de hacktivismo, sem necessariamente custos de compra. Este último requisito seria uma realidade impraticável quando comparado aos modelos tradicionais, seja pela criação artesanal ou pela produção comercial.

A produção de material pedagógico em formato digital é mais ágil e durável diante das produções artesanais comumente utilizada pelos professores, principalmente nas séries do ensino fundamental, além do formato digital ainda ser mais prático e barato para os alunos em geral, para outras pessoas interessadas e para estudantes com deficiências visuais também, na medida que podem ser executados com arquivos baixados abertamente, sem necessariamente estarem ligados à compra de alguns produtos.

Essa prática de substituir material pedagógico artesanal por artefato fabricado a partir de manufatura digital, com ênfase nos princípios da cultura maker, possui uma intencionalidade conceitual baseada no hacktivismo proposto por Samuel (2004), no sentido de que o desempenho dos hackers é caracterizado pelo uso não violento de ferramentas digitais para fins políticos. Desta forma, existe um propósito político anunciado neste trabalho de pesquisa que é a popularização do uso de software livre e hardware em favor da educação, aplicando material universal, aberto e replicável para pessoas, incluindo aquelas com deficiência visual. 
Nas seções a seguir serão apresentadas a forma como o projeto foi criado e desenvolvido, bem como os resultados obtidos até o momento.

Vários modelos foram formatados em laboratórios de inovação e suas características de sustentabilidade financeira vão desde aquelas em que os planos recebem incentivos governamentais, outras que fazem parte de estudos universitários públicos e privados, até aquelas propostas gerenciadas por associações civis, por exemplo.

Evidentemente esta linha de análise amplia as reflexões inclusive sobre a postura das próprias pessoas envolvidas. A inovação cidadã (IC) proposta pela Secretaria Geral Iberoamericana (SEGIB, 2019) é definida como um processo que apresenta soluções para problemas sociais apoiados por tecnologias (digital, social, ancestral) e metodologias inovadoras, buscando a participação da comunidade a quem a solução é abordada. Isso significa que os cidadãos deixam de ser receptores passivos de ações institucionais para se tornarem protagonistas e produtores de suas próprias soluções.

A pesquisa de caso científica apresentada neste trabalho foi realizada no Quinto Laboratório de Inovação Cidadã, organizado pela Secretaria Geral Ibero-Americana (Espanha), que anualmente promove laboratórios na América Latina, Portugal e Espanha.

Este laboratório ocorrido em 2018 contou com o apoio institucional da Secretaria de Inovação Pública do Governo da Província de Santa Fé (Argentina), Ministério da Modernização do Estado (Argentina), Fundação Ford (EUA), Centro Nansen para a Paz e Diálogo (Noruega), Internacional Espanhol Cooperação para o Desenvolvimento (Espanha) e Instituto Procomum (Brasil). A iniciativa contou com 10 projetos, num total de 100 cidadãos de 17 países que trabalharam em 23 comunidades locais.

Não é por acaso que os 10 temas de projetos aprovados e desenvolvidos em Rosário estão alinhados com os Objetivos de Desenvolvimento Sustentável (ODS / ONU). O laboratório foi 
intitulado "Comunidades Sustentáveis e Inclusivas". Aqui estão os temas dos projetos (INNOVACIÓN CIUDADANA, 2019):

- Elevações: cocriação de uma cadeira de pé com código aberto;

- Mulheres QOM capacitadas;

- Impressões 3D de modelos para aprendizado científico: este é o projeto abordado neste artigo;

- Sementes poderosas;

- Blockchain: a última fronteira da participação cidadã;

- Circuito autossustentado da aquaponia: peixes e plantas;

- Ocupação Beauvoir: onde estão os artistas?

- Troca digital sustentável;

- A rota trans: lugares seguros;

- Realidade virtual.

B. O processo de seleção, criação, compartilhamento e manutenção da popularização do conhecimento

O processo de seleção de projetos ocorreu em abril de 2018, a partir de uma chamada mundial. Dois aspectos principais destacam-se na linguagem e publicidade dada a este artigo: (1) foco especial em projetos que enfatizam a geração de maior coesão social, ou seja, aqueles projetos que buscam colaborar com sociedades que se unem em torno de um projeto comum de bem-estar, baseado em oportunidades de igualdade; equidade social e solidariedade, a fim de reduzir distâncias entre indivíduos, grupos e territórios, apoiados por novas tecnologias; (2) a condição de que os projetos selecionados sejam abertos à participação de outros colaboradores interessados que possam contribuir para o desenvolvimento científico e tácito durante o processo laboratorial.

O primeiro critério (1) mostra a característica de que a iniciativa tem foco na justiça social com suporte tecnológico, que é uma das principais contribuições de reflexão neste trabalho, 
enquanto a segunda regra (2) aponta a premissa de um recurso de design aberto, especialmente em relação à potencialidade de produzir materiais de compartilhamento digital durante o processo de criação do conhecimento.

Depois disso, ocorreu a seleção de colaboradores em maio de 2018. Foram escolhidos nove colaboradores de quatro diferentes países ibero-americanos. Perfis desejados foram estabelecidos a partir das habilidades e conhecimentos necessários para o desenvolvimento de projetos, incluindo: modelador digital com uso de software livre, programador em linguagens que utilizam código aberto, professores de educação especial, especialistas em hardware aberto, entusiastas e transformadores de diferentes especialidades (robótica, comunicação, eletrônica, design) e pessoas com deficiência visual.

Uma vez que a equipe foi selecionada, mesmo a distância, as articulações foram iniciadas por meio da troca de suas experiências anteriores e do início da criação de um repositório de informações de plataforma aberta. Mesmo conscientes da importância da cocriação do design de soluções físicas e digitais, provenientes do envolvimento da comunidade local, houve alguns desenvolvimentos para fundamentação do protótipo que viria a ocorrer em outubro de 2018, em Rosário.

Naquele mês, pouco antes do início das atividades do laboratório, a equipe local de apoio e articulação (Santa Lab) buscou e se aproximou de três instituições que demonstraram interesse em ter suas necessidades patrocinadas pelo projeto, foram elas: o Departamento de Educação da província local representado por professores e alunos com deficiência visual da Escola Especial 2081 - Lidia Elsa Rousselle; uma associação civil que visa garantir os direitos das pessoas com deficiência visual (Movimiento de Unidad de Ciegos y Ambliopes de Rosário - MUCAR); e o Aquário do Rio Paraná, com grande interesse na criação e implantação de material acessível para pessoas com deficiência visual, já que uma experiência em um aquário é estritamente visual e pouco sensorial. 
Tomando como critério principal o número de pessoas que poderiam ser beneficiadas pela realização, ou seja, o impacto do protótipo, o Departamento de Educação da província local foi escolhido como campo de aplicação do projeto, assim como para o uso do material pedagógico a ser cocriado.

Nesse ponto, a abordagem digital e educacional em si começou a se concentrar na ação de expandir a alfabetização digital dos professores e professoras da cidade de Rosário / Argentina que participariam desse projeto.

Assim, considera-se oportuno mencionar que a alfabetização digital, na definição de Souza (2007), constitui uma série complexa de valores, práticas e habilidades localizadas social e culturalmente envolvidas no funcionamento linguístico dentro de um contexto de ambientes eletrônicos, incluindo leitura, escrita e comunicação.

Somando-se a essa definição, o conceito maker, de Pinzeta \& Frosch (2019), reforça que, além de fazer, experimentar e compartilhar, a cultura maker se coloca como oportunidade metodológica para abordar práticas, bem como uma forma de organizar parte do conteúdo curricular, foram construídos materiais pedagógicos tanto em trajetórias formais quanto tácitas, sob a lógica da Ética do Cuidado.

Desta forma, foi preponderante que os três professores eleitos para a experiência tivessem um perfil receptivo para a aprendizagem digital, para o desenvolvimento e para o envolvimento com as tecnologias abertas para modelagem e impressão $3 \mathrm{D}$, pois estrategicamente já se comprometeram a um compromisso posterior de disseminar as aprendizagens da cultura digital para a rede de professores locais da rede pública, com pleno domínio conceitual e prático dos aspectos aprendidos durante as práticas de laboratório.

O programa de aprendizado digital passou pelos seguintes aspectos formativos: práticas e aplicações dos princípios makers contidos no Manifesto Maker (HATCH, 2017); reconhecimento, aprofundamento e implementação prática das questões do hacktivismo na educação especial (open source, compartilhamento digital, autoprodução de elementos impressos em 3D e apli- 
cação pedagógica); busca e manipulação de arquivos STL em plataformas abertas. Além desses, possibilitou acesso ainda a informações sobre apresentação e uso de fundamentos básicos de modelagem digital com aprendizado de softwares livres; reconhecimento da tecnologia de impressão Fused Deposition Modeling (FDM) e propriedades dos materiais consumíveis no processo de impressão com PLA e ABS; conceituação e aplicação de fatiamento de modelo digital e geração de linguagens em protocolo g-code para impressão em hardware livre; criação de perfis e upload de modelos criados a partir de situações reais de currículos pedagógicos; estruturação de tutoriais e estratégias para disseminar conteúdo para outros professores.

A perspectiva epistemológica pode ser ilustrada pelo fluxo publicado por Pinzeta \& Frosch (2019):

Figura 1: Fluxo metodológico de produção maker de recurso pedagógico.

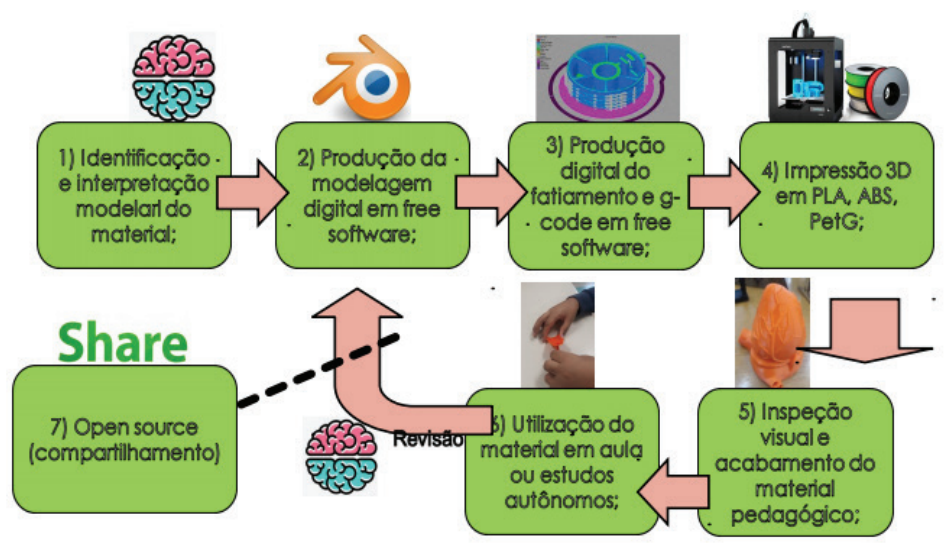

Fonte: Pinzeta \& Frosch (2019).

O ponto crucial para o sucesso do protótipo do material pedagógico foi a definição de fabricação digital a partir do conteúdo curricular escolhido pelas próprias professoras, ou seja, uma escolha reflexiva e assertiva do primeiro campo do fluxo 
“identificação e interpretação modelar do material", como pode ser observado nas figuras a seguir:

Figura 2: modelo existente no site Thingiverse
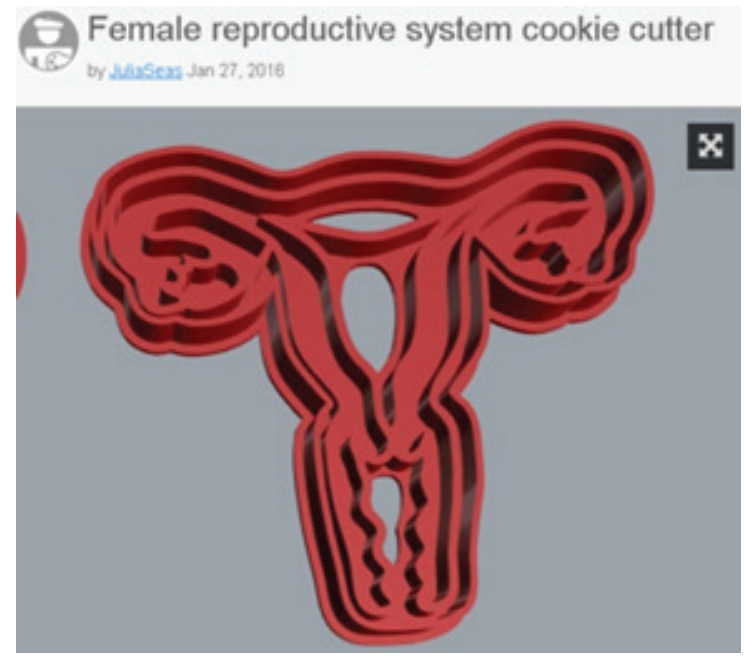

Fonte: Thingiverse (2019)

Figura 3: Modelo criado e compartilhado digitalmente fe. Female Reproductive System

by tusich Oet 30,2018

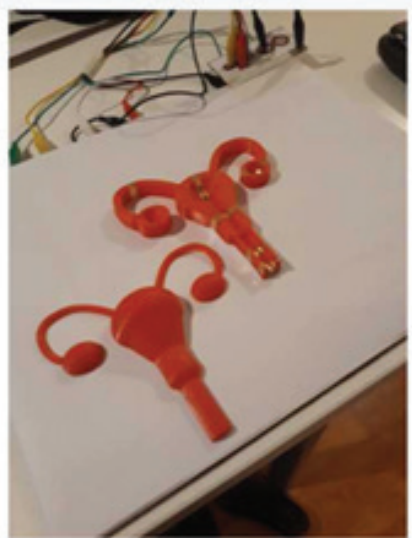

Fonte: Thingiverse (2019) 
Os princípios do material didático se basearam no conhecimento prático de professores de escolas especiais, como tamanhos, texturas, cores, formas. Essas percepções foram somadas às experiências do professor colaborador com deficiência visual e, por fim, articuladas aos conteúdos tecnológicos trazidos pelos demais integrantes da equipe do projeto.

Acredita-se que o material pedagógico utilizado no processo educativo para pessoas com algum tipo de deficiência visual tem uma série de especificidades que necessitam ser analisadas. Esse aspecto nos remete a Cerqueira e Ferreira (2000), que apontam que a especificidade desse processo de aprendizagem está baseada nos seguintes aspectos:

- Um dos problemas básicos do deficiente visual é a dificuldade de contato com o ambiente físico;

- A carência de material adequado pode conduzir a aprendizagem da pessoa com deficiência visual a um mero verbalismo, desvinculado da realidade;

- A formação de conceitos depende do íntimo contato do indivíduo com as coisas do mundo;

- Tal como a pessoa de visão normal, o deficiente visual necessita de motivação para a aprendizagem;

- Alguns recursos podem suprir lacunas na aquisição de informações pelo deficiente visual;

- O manuseio de diferentes materiais possibilita o treinamento da percepção tátil, facilitando a discriminação de detalhes e suscitando a realização de movimentos delicados com os dedos.

O conteúdo curricular escolhido foi a modelagem do trato reprodutivo feminino, pois era um material possível para antecipar a produção, conforme exigido pelo planejamento acadêmico, bem como algo inédito em 3D nas plataformas de arquivos digitais abertos até aquele momento. Encontramos apenas um modelo $2.5 \mathrm{~d}$, uma expressão adotada por professores de educação especial quando eles querem se referir a modelos 
que experimentam apenas extrusões em baixa altura no eixo $\mathrm{Z}$, como o exemplo evidenciado na Figura 2.

\section{COMENTÁRIOS FINAIS}

Lafuente (2014, p.16) afirma que em um momento como o contemporâneo, em que se investiga sobre o comum, o contexto desenhado parece clamar por nós para explorar as relações intrincadas e inescapáveis entre o público, o privado e os comuns no contexto particular da produção: que relações de dependência, contiguidade e exclusão existem entre o público e os comuns? Existem formas de patrimonialização que contribuem para a geração de bens comuns? E um segundo aspecto se refere à relação entre os bens comuns e a cultura livre (ou tecnologias livres) da qual as contribuições deixam um registro.

Independentemente das fortes correntes individualistas que enfrentamos hoje, as práticas colaborativas, especialmente na América Latina, persistem e surgem apesar da falta de apoio dos governos, porque estão enraizadas na cultura do trabalho voluntário em comunidades ou em grupos específicos para fins de utilidade social, ou aqueles ancorados em natureza recíproca que usam tecnologia disponível com ênfase em processos baseados nos princípios da lógica do comum.

\section{AGRADECIMENTOS}

Agradecimentos aos membros da equipe do projeto Con Tacto 3D, à Secretaria Geral Ibero-Americana (SEGIB - Espanha), nas figuras de Mariana Cancela e Pablo Pascale; e ao SantaLab (Argentina), na pessoa de Dardo Ceballos, pelo apoio durante o período de produção do projeto.

\section{REFERÊNCIAS}

CERQUEIRA, Jonir Bechara; FERREIRA, Elise de Melo Borba. Recursos didáticos na educação especial. Revista Brasileira Para Cegos: Instituto Benjamin Constant, Rio de Janeiro, v. 516, p. 12-14. Trimestral. Abr. 2000. 
GERSHENFELD, Neil. Fab: The Coming Revolution on your Desktop - from Personal Computers to Personal Fabrication. EUA. New York: Basic Books, 2005.

HATCH, Mark. The maker movement. Manifesto. Disponível em: < http:/ / www.techshop.ws/images/0071821139 Maker Movement Manifesto Sample Chapter.pdf> Acesso em: 03 set. 2017.

INNOVACIÓN CIUDADANA - IC. Proyectos del LABICAR. Disponível em https:/ /www.innovacionciudadana.org/laboratorios/proyectos-del-labicar-rosario-2018/ Acesso em: 03 abril de 2019.

LAFUENTE, Antonio; ESTALELLA, Adolfo. Modos de ciencia: pública, abierta y común. In: ALBAGLI, S.; MACIEL, M. L. ; ABDO, A. H. (Org.). Ciência aberta, questões abertas. Brasília: Ibict; Rio de Janeiro: Unirio, 2015.

LAFUENTE, Antonio; ESTALELLA, Adolfo. Borrador de capítulo para el libro: Teknokultura entre dos siglos. Tecnociencia, arte y cultura. Laboratorios de procomún. Madrid: Catarata, 2014.

PINZETA, Priscila; FROSCH, Renato. Produção maker de material pedagógico com impressora 3d para pessoas com deficiência visual. In: MELO, Douglas Christian Ferrari de; PEROVANO, Laís Perpétuo (Org.). Práticas inclusivas: saberes, estratégias e recursos didáticos, cap. 8. Campos dos Goytacazes/ RJ: Brasil Multicultural, 2019.

SAMAGAIA, Rafaela; DELIZOICOV NETO, Demétrio. ENCONTRO NACIONAL DE PESQUISA EM EDUCAÇÃO EM CIÊNCIAS, 2015. Educação científica informal no movimento "Maker". Águas de Lindóia: Enpec, 2015.

SAMUEL, Alexandra Whitney. Hacktivism and the Future of Political Participation. Cambridge: Harvard University, 2004.

SECRETARIA GENERAL IBEROAMERICANA - SEGIB. Laboratorios de innovación ciudadana ¿Qué son y para qué? Disponível em https://www. segib.org/laboratorios-de-innovacion-ciudadana-que-son-y-para-que/ Acesso em: 03 abril de 2019.

SOUZA, V. V. Soares. Letramento digital e formação de professores. Revista Língua Escrita, n. 2, dez. 2007. 


\section{SOBRE O AUTOR:}

\section{RENATO FROSCH}

É maker; doutorando em Educação, pela Universidade Católica de Santos UNISANTOS; professor universitário nos cursos de Pedagogia, Engenharia, Arquitetura e Administração; em 2018, foi promotor de projeto no Laboratório de Inovação Cidadã, em Rosário, Argentina.

E-mail: prof.renatofrosch@gmail.com 